\title{
Quality by Design (QbD) como ferramenta para otimização dos processos farmacêuticos
}

\author{
Quality by Design (QbD) as a tool for optimization \\ of pharmaceutical processes.
}

Recebido em: 28/09/2016 Aceito em: $03 / 03 / 2017$
Mariana Palmeira BEZERRA; Leticia Norma Carpentieri RODRIGUES Departamento de Ciências Farmacêuticas, Instituto de Ciências Ambientais, Químicas e Farmacêuticas, Universidade Federal de São Paulo. Rua São Nicolau, 210 Diadema, SP. CEP 09913-030. Brasil.E-mail: leticia.carpentieri@gmail.

\section{ABSTRACT}

The pharmaceutical industry is constantly evolving. The harmonization of production standards, with the aim of guaranteeing efficacy, safety and quality of medicines, is one of the greatest challenges. The concept of "Quality by design" (QbD) proposes a systematic approach, based on scientific knowledge and risk management associated with the manufacturing process. In this approach, quality is inversely proportional to variability. The implementation of the QbD concept is a promising tool for pharmaceutical production, since it allows the production of medicines by means of risk prediction, increasing the possibility of generating products with efficiency, safety and quality assured, together with the reduction of costs. The implementation of this concept requires not only new technologies, but the change in the concept of quality.

Keywords: pharmaceutical processes; quality by design; process analytical techniques.

\section{RESUMO}

O setor farmacêutico está em constante evolução. A harmonização de normas de produção, com o intuído de garantir eficácia, segurança e qualidade dos medicamentos, consiste em um dos maiores desafios. O conceito "qualidade baseada no projeto" (Quality by design, QbD) propõe uma abordagem sistemática, fundamentada no conhecimento científico e no gerenciamento do risco associado ao processo de fabricação. Nesta abordagem, qualidade é inversamente proporcional a variabilidade. A implementação do conceito de $Q b D$ constitui uma ferramenta promissora para a produção farmacêutica pois permite a produção de medicamentos por meio da previsão de riscos, ampliando a possibilidade de gerar produtos com eficácia, segurança e qualidade assegurados, aliado a redução de custos. A implementação deste conceito exige não somente novas tecnologias, mas a mudança no conceito de qualidade.

Palavras-chave: processos farmacêuticos; qualidade baseada no projeto; técnicas analíticas de processo

\section{INTRODUÇÃO}

A produção de medicamentos inclui uma série de processos farmacêuticos, caracterizados por uma sequência de operações unitárias, que realizam transformações químicas ou físicas em matérias-primas, com o fim de gerar produtos com eficácia, segurança e qualidade assegurados (1). Estabelecidos durante o desenvolvimento dos produtos, os processos farmacêuticos devem ser submetidos a aprovação por órgãos regulatórios para concessão de registro de comercialização, o qual assegura que a empresa comprovou ter condições de fornecer produtos de qualidade desejada de forma consistente, conforme as exigências regulatórias $(2,3)$.

Apesar do rigor das agências regulatórias na elaboração de normas e guias para a produção farmacêutica, estes requerimentos apresentam limitações, e falhas nos processos farmacêuticos são comumente observadas, o 
que resulta em modificações de registro, desabastacimento de produtos e, principalmente, impacto na saúde pública $(4,5)$.

O termo Quality by Design, em português traduzido para "Qualidade baseada no projeto" e atualmente conhecido pela sua sigla no idioma original - $Q b D$, foi criado em meados da década de 1950, pelo engenheiro Joseph Moses Juran, o qual suportava a ideia de que os problemas referentes à qualidade de um produto estão relacionados ao modo com que ele foi desenvolvido e, portanto, a qualidade deve ser controlada a cada etapa do processo para maximizar a probabilidade de que o produto final alcance seus atributos de qualidade e as especificações (6).

A Garantia da Qualidade se resume a totalidade das providências tomadas com objetivo de garantir que os medicamentos estejam dentro dos padrões de qualidade exigidos pelo mercado e pelos órgãos reguladores, para que possam ser utilizados com segurança pela população. O Gerenciamento de Riscos é um processo essencial que permite a indústria garantir a qualidade dos produtos.

Gerenciamento de Riscos é o processo de planejar, organizar, dirigir e controlar os recursos humanos e materiais de uma organização, no sentido de minimizar os efeitos dos riscos sobre essa organização, ao mínimo possível. O risco simboliza a incerteza quanto à ocorrência de um determinado evento com potencial para causar danos. Esses danos podem ser entendidos como lesões a pessoas, danos a equipamentos e instalações, danos ao meio ambiente, perda de material em processo, ou redução da capacidade de produção. É praticamente impossível a eliminação completa de todos os riscos. A avaliação dos riscos deve ser embasada em conhecimento científico, e o nível de esforços, formalidade e documentação do processo de gerenciamento de riscos deve ser adequado ao grau do risco do produto.

Introduzido na Industria Farmacêutica pela agência regulatória americana (Food and Drug Administration) por meio dos documentos Pharmaceutical Control Good Manufacturing Practices (cGMPs) for the $21^{\text {th }}$ Century (7) e, Guidance for Industry Process Analytical Technology (PAT) (8), o conceito $Q b D$ atualmente está sendo conduzido pela Conferência Internacional de Harmonização dos Requisitos Técnicos para Registro de Produtos Farmacêuticos para Uso Humano (International Council for Harmonisation of Technical Requirements for Pharmaceuticals for Human Use, ICH), entidade onde participam as agências regulatórias ame- ricana (Food and Drug Administratiom, FDA), europeia (European Medicines Agency, EMA) e japonesa (Japan Pharmaceuticals and Medical Devices Agency, Japan $P D M A)$. O documento técnico ICH Q8(R2) (9) fornece as diretrizes para implementação do $Q b D$ e, mais recentemente, o ICH Q11 (10) contempla os conceitos de validação e verificação continuadas ao longo do ciclo de vida do produto.

A Conferência Internacional de Harmonização $(\mathrm{ICH})$, por meio destes documentos técnicos, busca definir uma nova concepção na produção de medicamentos, baseada não apenas nos testes finais realizados no produto, mas na aplicação dos princípios do Quality by Design, consistindo em uma abordagem sistemática do desenvolvimento farmacotécnico, que inicia com objetivos pré-definidos e dá ênfase à compreensão do produto, do processo e do controle de qualidade deste, com base na ciência e no gerenciamento de riscos de qualidade.

A implementação do conceito $Q b D$ envolve "elementos" e "ferramentas". Os elementos da $Q b D$ buscam direcionar os objetivos do processo, demonstrar como um produto deve ser descrito sob a perspectiva das necessidades do paciente, e definir os atributos que permitem a investigação experimental; as "ferramentas" do $Q b D$ visam o monitoramento da qualidade por meio do controle sistemático das variáveis envolvidas no processo $(9,11)$.

\section{Os “ELEMENTOS” da Quality By Design (QbD)}

"Perfil do Produto" (Quality target product profile, QTPP). O "Perfil do Produto" (Quality target product profile, QTPP) é representado pelo conjunto de atributos do produto relacionados às demandas do paciente: via de administração, dose, sistema de liberação, etc $(9,12,13)$. Apesar do $Q T P P$ ser semelhante às especificações do produto presentes no modelo tradicional de qualidade, no contexto da $Q b D$ esses atributos ganham novo significado e função, pois representam os atributos do produto final que definem sua eficácia e segurança terapêutica (6).

“Atributos Críticos da Qualidade" (Critical quality atributes, $C Q A)$. Os "Atributos Críticos da Qualidade" ( $C Q A)$ são representados pelas propriedades químicas, físicas, biológicas e/ou microbiológicas e visam garantir que o produto tenha a qualidade desejada (9). Os $C Q A$ podem pertencer ao produto final, intermediários ou as matérias-primas, e têm impacto significativo na qualidade do produto final (14). Os $C Q A$ determinam o "Perfil do Produto" $(Q T P P)$ e, portanto, estão intima- 
mente relacionadas com o desempenho clínico do produto (15). No desenvolvimento do produto por meio da abordagem $Q b D$, os $C Q A$ são critérios de qualidade que podem ser medidos, e assim correlacionados experimentalmente às variáveis do processo. Neste contexto, é relevante observar que se fossem considerados todos os $C Q A$ de um processo, estes seriam um obstáculo a fase experimental da investigação devido ao grande número de experimentos para comprovar a relação entre eles e as varáveis do processo. O documento técnico $I C H \mathrm{Q} 8(\mathrm{R} 2)$ (9) destaca a necessidade da utilização de "ferramentas" de gerenciamento de riscos para priorizar os $C Q A$ mais relevantes no planejamento dos experimentos.

"Atributos Críticos do Material" (Critical material atributes, $C M A)$. Os "Atributos Críticos do Material" $(C M A)$, representado pelas matérias-primas, são uma extensa fonte de variação no processo de fabricação. O controle dos CMA é essencial para a garantia da qualidade do produto, e seu impacto é facilmente identificado no produto final quando não controlados (9). A importância do $C M A$ no $Q b D$ é ressaltar a importânica da funcionalidade das matérias-primas no desenvolvimento de uma formulação. A abordagem $Q b D$ complementa a perspectiva tradicional de qualidade que considera as matérias-primas por características de identificação, e negligencia as suas propriedades físicas ou funcionais, cuja variabilidade pode alterar o processo farmacêutico e afetar a qualidade dos produtos finais $(9,13)$. Um processo farmacêutico contém inúmeras $C M A$, isto porque uma formulação é composta por vários componentes e cada um destes apresenta ínumeros tipos de atributos. Nesse contexto, a avaliação de riscos atua como um facilitador, pois indica quais os CMA exigem maior atenção, reduzindo o gasto de recursos disponíveis para estudo $(9,11)$. As matérias-primas, quando submetidos a determinadas operações unitárias, podem sofrer alterações determinantes no desempenho clínico do produto. Um exemplo é a transformação polimórfica, comumente observada em algumas substâncias ativas, decorrente de mudanças mínimas de temperatura e/ou pressão, ou outros fatores. Polimorfos apresentam propriedades físicas e químicas distintas, tal como a solubilidade, que tem impacto sobre o perfil de dissolução e biodisponibilidade do fármaco e, portanto, afetam a eficácia do produto (16).

"Parâmetros Críticos do Processo" (Critical Process Parameters, CPP). No processo, quando um parâmetro tem papel significativo na qualidade do material fabricado, este é identificado pela abordagem $Q b D$ como um "Parâmetro Crítico do Processo" e deve ser avaliado quanto ao risco que sua variação representa na qualidade do produto final (9). Um processo envolve muitas variáveis relacionadas a operação dos equipamentos tais como a frequência de rotações, a velocidade de aquecimento, o fluxo, a temperatura de secagem, etc (14). Os "elementos" da abordagem $Q b D-C Q A, C M A$ e $C P P$ - são essenciais para estruturar o processo, e assim facilitar o seu desenvolvimento e aperfeiçoamento. Yu e cols (2014) mostrarama relação entre esses três elementos por meio de uma função matemática (11) (Figura 1).

"Espaço do Projeto" (Design Space, DS). O "Espaço do Projeto" consiste em um espaço virtual onde são estabelecidas combinações e/ou interações multidimensionais das variáveis de um processo e de seus parâmetros, visando proporcionar a garantia de qualidade. Segundo a $F D A$ o $D S$ deve ser determinado a partir da combinação de, pelo menos, três variáveis, sendo o planejamento experimental (Design of Experiments, DoE) recomendado para alcançar esses objetivos. O "Espaço do Projeto" $(D S)$ é o descritivo de um conjunto de variáveis que, ajustadas a limites conhecidos, garantem a qualidade do produto final. Uma vez demonstrada uma trajetória racional no desenvolvimento, o $D S$ pode ser descrito por uma combinação de limites operacionais ou por sofisticados modelos matemáticos. A abrangência do $D S$ pode ser aplicada, separadamente, para cada unidade de operação, ou pode representar todo o processo, sendo que a escolha determina o grau de complexidade dos experimentos, e tem impacto na flexibilidade da operação dentro do espaço (9).

Em comparação com o método tradicional - um-fator-a-um-tempo - (One Fator At a Time, OFAT), o planejamento experimental (Design of experiments, $D o E$ ) avalia as variáveis e suas interações, reduzindo drasticamente o número de experimentos. Do ponto de vista prático, o $D S$ se assemelha às condições normais de operação da abordagem tradicional, no entanto, sob uma perspectiva mais flexível $(13,14)$. No modelo tradicional, a abordagem de "intervalos aceitáveis comprovados" (proven acceptable ranges, PAR) baseia-se na definição dos parâmetros críticos do processo e dos intervalos dentro dos quais eles devem ser controlados para garantir que o produto atenda aos seus atributos de qualidade predeterminados; o $D S$, por outro lado, se concentra na definição dos parâmetros críticos do processo que, devidamente controlados, garantirão que o produto atenda às especificações pré-determinadas e atributos de qualidade. Os órgãos regulatórios estabeleceram que modificações na operação do processo dentro do "Espaço do Projeto" $(D S)$ delimitado não exige alteração do registro após sua concessão $(9,17)$. 


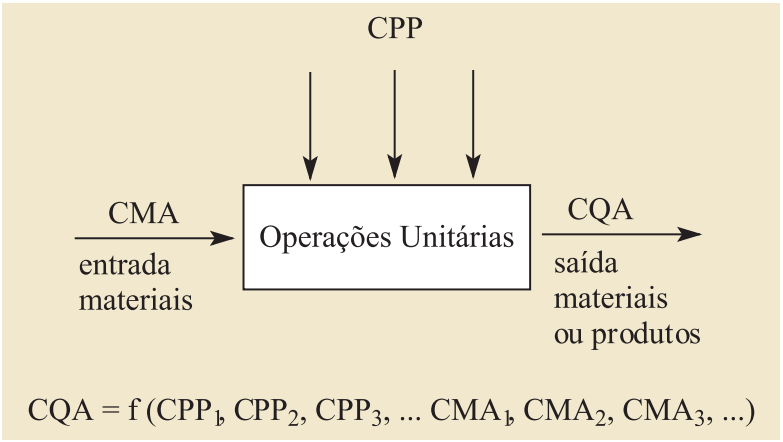

Figura 1: Relação entre as variáveis do processo e os atributos críticos de qualidade. Adaptado de Yu e cols, 2014 (11)

\section{As "FERRAMENTAS" da Quality By Design}

"Planejamento Experimental" (Design of Experiments, DoE). O Planejamento Experimental (DoE) tem como objetivo fornecer informações dos dados experimentais considerando, racionalmente o sistema e os recursos disponíveis. Os experimentos devem ser planejados como base em seu objeto de estudo, onde se investigam os fatores mais importantes entre um conjunto de variáveis pouco conhecidas. Os estudos empregando $D o E$ agregam duas fases de investigação: em uma primeira fase as principais fontes de variação são identificadas por meio do DoE exploratório (screening); em seguida, os resultados desses experimentos iniciais são empregados nos estudos das interações entres os fatores do sistema - $D o E$ de otimização $(18,19,20)$. O "planejamento experimental" ( $D o E)$ é realizado por meio de programas específicos (Design Expert, MatLab, JMP, SIMCA, entre outros) que se diferenciam pela inteface, opções de desenho e disponibilidade de ferramentas de análise de dados $(21,22)$. Estes produtos também auxiliam a determinar o modelo que melhor representa o objeto de investigação por meio de tratamento estatístico $(21,23)$.

"Avaliação do Risco" (Risk Assessement, RA). A "Avaliação do Risco" $(R A)$ permite discriminar as fontes de variação de um sistema e, consequentemente, priorizar os pontos de investigação de um processo. O ICH Q9 (24) sugere o emprego de modelos de $R A$ tais como o "Diagrama de Ishkawa", a "Análise preliminar de perigo" (Preliminary Hazard Analysis, PHA), a "Análise da árvore de falhas" (Faut tree analysis, FTA), o mapeamento de risco, o "Estudo de perigo e operabilidade" (Hazard and operability study, HAZOP), a filtração e priorização de risco (Risk Ranking and Filtering) e, mais recentemente, a "Análise de criticidade e modo de efeito de falhas" (Failure Mode and Effects Analysis, FMECA) $(12,19)$, uma técnica que analisa os potenciais modos de falhas de um processo e de seus efeitos com relação à confiabilidade e segurança. De modo geral, a condução de uma $R A$ depende da profundidade do conhecimento que se tem do objeto de estudo $(12,19,24)$.

A Figura 2 apresenta o emprego do "Diagrama de Ishkawa" como ferramenta para o desenvolvimento de Formas Farmacêuticas Sólidas Orais (FFSO). As seis fontes de variação de um processo: o ambiente, as propriedades da matéria-prima, as condições de operação do processo (granulação, secagem e compressão) e as técnicas analíticas empregadas no controle de qualidade estão representadas no modelo (9).

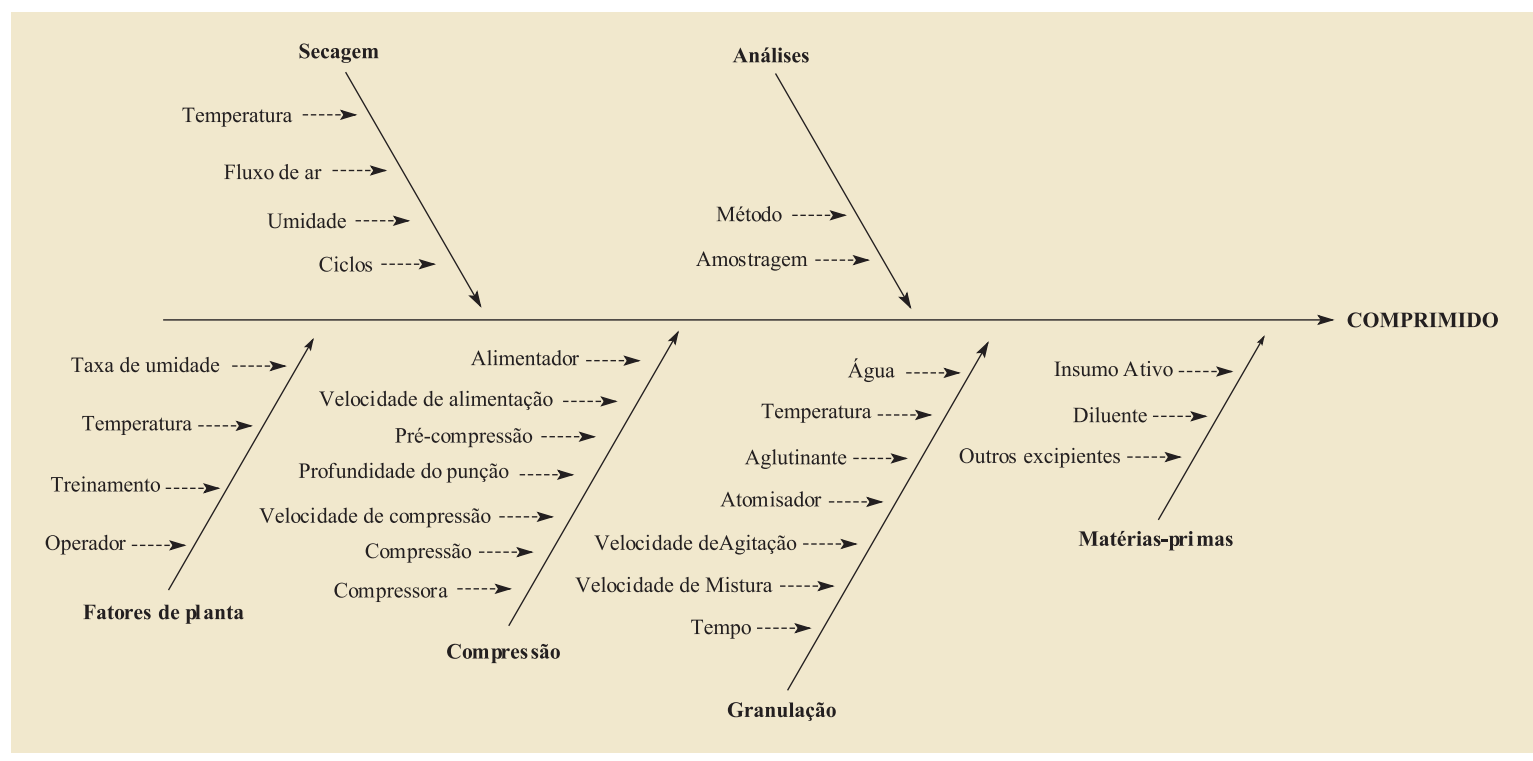

Figura 2: Diagrama de Ishikawa. Desenvolvimento de FFS. Adaptado do ICH Q8(R2) (9) 
A $R A$ é realizada ao longo do processo de desenvolvimento do produto. Tem início por meio do conhecimento agregado a partir de experiências anteriores, devendo ser aperfeiçoada com base nas informações obtidas nos estudos experimentais $(9,19)$. Para que a $R A$ seja efetiva, é recomendado que a sua elaboração tenha a colaboração de especialistas relacionados ao objeto de estudo, para que o sistema seja avaliado da forma mais abrangente e acurada. A execução dessa análise em grupos de trabalho assegura o correto direcionamento dos experimentos das variáveis de proces-

Quadro 1: Representação esquemática da "Avaliação de riscos" Adaptado da Food and Drug Administration (25)

\begin{tabular}{|c|c|c|c|c|c|}
\hline \multirow{2}{*}{$\begin{array}{l}\text { Atributos Críticos do } \\
\text { Processo } \\
(C Q A)\end{array}$} & \multicolumn{5}{|c|}{ Etapas do Processo } \\
\hline & $\begin{array}{l}\text { Mistura e } \\
\text { Lubrificação }\end{array}$ & $\begin{array}{l}\text { Compressão } \\
\text { (Pastilhão) }\end{array}$ & Moagem & $\begin{array}{l}\text { Mistura Final e } \\
\text { Lubrificação }\end{array}$ & $\begin{array}{l}\text { Compressão } \\
\text { (Comprimido) }\end{array}$ \\
\hline Ensaio & Médio & Baixo & Médio & Baixo & Médio \\
\hline Uniformidade de conteúdo & Alto & Alto & Alto & Baixo & Alto \\
\hline Dissolução & Médio & Alto & Médio & Alto & Alto \\
\hline Produtos de Degradação & Baixo & Baixo & Baixo & Baixo & Baixo \\
\hline
\end{tabular}

Uma das limitações da $R A$ é quantificação do risco, uma vez que os métodos empregados para a avaliação de riscos são normalmente qualitativos ou apresentam escalas subjetivas. Uma forma de aumentar a clareza da $R A$ é realizar experimentos que permitem relacionar as variáveis do processso com as características de qualidade do produto por meio do "planejamento experimental" (DoE) (19).

"Técnicas Analíticas de Processo" (Process Analytical Techniques, PAT). As "Técnicas analíticas de processo" (PAT) são um conjunto de ferramentas capazes de dar suporte ao desenvolvimento, produção e controle de qualidade do produto, avaliando e reduzindo os riscos (8).

As PAT são descritas em categorias segundo seu papel no sistema de qualidade: ferramentas estatísticas aplicadas ao planejamento de experimentos, aquisição e análise de dados; analizadores de processos; ferramentas de controle de processo; e métodos de gestão do conhecimento (8).

Inseridas em equipamentos empregados no processo, as $P A T$ permitem que dados qualitativos e quantitativos da amostra sejam registrados, e comparados com um grande conjunto de dados experimentais previamente obtidos e tratados por meio de análise multivariada de dados $(16,26,27,28,29,30)$ e, a presença de não confor- so (18). Uma ferramenta de avaliação amplamente difundida é a "Análise de criticidade e modo de efeito de falhas" (Failure Mode and Effects Analysis, FMECA), onde um cenário de falha das operações do processo é suposto e procura-se quantificar o impacto da falha na qualidade do produto. De modo geral, a atribuição de intensidade do risco considera o produto da multiplicação de três fatores: a frequência de falha (probabilidade), a severidade do efeito da falha (severidade), e a capacidade de detectar a falha (detectabilidade) (18) (Quadro 1).

(RA) nas diversas etapas do processo de compressão via seca. midade, permite a intervenção na linha de produção para ajustes e correção de riscos. A aquisição de dados na linha de produção, por meio das PAT agrega informações ao processo, possibilitando o aperfeiçoamento contínuo (16,31). O avanço das $P A T$ representa uma grande inovação na indústria farmacêutica, pois amplia a capacidade de monitoramento dos processos e altera radicalmente a forma de execução do controle de qualidade. Por meio das $P A T$, a qualificação da matéria-prima ocorre na sua embalagem original, sem a necessidade de amostragem ou ainda áreas exclusivas para armazenamento e ensaios de qualidade; paralelamente, os métodos tradicionais de controle de qualidade do produto final perdem espaço para as $P A T$ as quais permitem o monitoramento do processo de forma contínua e em tempo real $(16,30)$. A proposta de liberação dos dados em tempo real representa uma mudança estrutural do processo do produtivo, porque insere o controle de qualidade na linha de produção $(9,32,33)$.

A espectroscopia de infravermelho próximo (Near-infrared spectroscopy, NIR) e espectroscopia Raman são "Técnicas Analíticas de Processo", não-destrutivas, amplamente empregadas para o controle do processo farmacêutico em tempo real dentro da abordagem da $Q b D$. Parâmetros como uniformidade de conteúdo, teor, umidade, monitoramento da operação de mistura, espessura 
de revestimentos, etc são obtidos em tempo real permitindo a garantia da qualidade do produto $(16,18,31)$.

O emprego de PAT reduz o tempo de residência do produto na planta de fabricação, no que se denomina "Liberação em Tempo Real", constituindo um novo conceito de qualidade $(3,8,9,14,32)$.

\section{Quality by design $(Q b D)$ e as agências regulatórias}

A implementação da abordagem $Q b D$ representa uma mudança na forma de produção da indústria farmacêutica, onde as operações unitárias estão fisicamente conectadas, desde a alimentação das matérias-primas até a obtenção do produto final, de forma contínua. Um processo contínuo exige equipamentos específicos, menores que os tradicionais, o que não reduz a sua capacidade produtiva, já que a determinação do volume de produção passa a depender do tempo de operação transcorrido e não do tamanho dos equipamentos. A principais vantagens do processo contínuo são a redução dos custos e o tempo de desenvolvimento do processo, uma vez que a fase de transferência de escala torna-se desnecessária nesse novo conceito de produção (30).

A implementação da abordagem $Q b D$ facilita o trabalho de revisão das solicitações de registro pelas agências regulatórias, já que destaca, de forma estruturada, os pontos que comprovadamente oferecem risco a qualidade do produto $(6,34,35)$.

A comunidade científica tem reforçado a necessidade do emprego da abordagem $Q b D$ no desenvolvimento de produtos $(4,5,34)$. O comitê gestor da Conferência Internacional de Harmonização (ICH) enfatizou a ine- xistência de obrigatoriedade no cumprimento das diretrizes estabelecidas nos guias, entretanto alertaram que as recomendações refletem as perspectivas das agências regulatórias sobre a qualidade dos produtos farmacêuti$\cos (3,9,24)$.

Apesar dos esforços do ICH e da comunidade científica, a adesão à abordagem $Q b D$ na indústria farmacêutica é lenta e apresenta resistências. A maior barreira na implementação da abordagem $Q b D$ é o investimento necessário para o desenvolvimento, validação e manutenção das bibliotecas espectrais de referência, adequação dos equipamentos de processo, e a instalação de ferramentas de análise $(4,5,34)$.

\section{CONCLUSÃO}

A agências regulatórias, impulsionadas pela inciativa do $F D A$, lançaram um novo paradigma de qualidade com base no conhecimento científico e gerenciamento de riscos associado a qualidade, o Quality by Design $(Q b D)$.

Os pontos fundamentais dessa abordagem são as ferramentas de estruturação do conhecimento do processo, que possibilitam que todas a variáveis relevantes a qualidade sejam identificadas, e que estratégias de controle sejam implementadas ao processo.

A implementação da $Q b D$ no desenvolvimento de produtos e aperfeiçoamento de processos é necessário por aumentar a eficiência do setor farmacêutico, atualmente pressionado pelo custo de operação, pela competitividade do setor e pela necessidade de lançamento de novos produtos que sustentam os investimentos.

\section{REFERÊNCIAS}

1. Lachman L, Hanna SA, Lin, K. In: Lachman L, Lieberman HA, Kanig JL. Teoria e prática na indústria farmacêutica. Lisboa: Fundação Calouste Gulbenkian; 2001. p.1357-1440.

2. BRASIL. Agência Nacional de Vigilância Sanitária (Anvisa). Resolução no 17 de 16 de abril de 2010 que trata das "Boas Práticas de Fabricação de Medicamentos". Disponível em: http://189.28.128.100/dab/docs/legislacao/resolucao17_16_04_10.pdf. Acesso em: $11 \mathrm{dez}$ 2016.

3. ICH. International Conference on Harmonisation of Technical Requirements for Registration of Pharmaceuticals for Human Use. ICH Harmonised Tripartite Guideline. Pharmaceutical Quality System Q10. 04 June
2008. Disponível em: http://www.ich.org/fileadmin/Public_Web_Site/ICH_Products/Guidelines/Quality/Q10/ Step4/Q10_Guideline.pdf. Acesso em: 14 nov 2016.

4. Aksu B, Beer T, Folestad S, Ketolainen J, Lindén H, Lopes JA, Matas M, Oostra W, Rantanen J, Weimer M. Strategic funding priorities in the pharmaceutical sciences allied to Quality by Design (QbD) and Process Analytical Technology (PAT). Eur J Pharm Sci. 2012; 47(2):402405. DOI: 10.1016/j.ejps.2012.06.009.

5. FDA. Department of Health and Human Services Food and Drug Administration. Center for Drug Evaluation and Research (CDER). Advancement of Emerging Technology Applications to Modernize the Pharmaceutical Manufacturing Base Guidance for Industry. December 
2015. Disponível em: http://www.fda.gov/downloads/ Drugs/GuidanceComplianceRegulatoryInformation/ Guidances/UCM478821.pdf. Acesso em: 14 dez 2016.

6. Garcia Aponte OF, Vallejo Diaz BM, Mora Huertas CE. La calidad desde el diseño: principios y oportunidades para la industria farmacéutica. Estudios Gerenciales. 2015; 31(134):68-78. DOI: 10.1016/j.estger.2014.09.005.

7. FDA. Department of Health and Human Services. Food and Drug Administration. Harmaceutical cGMPS for the $21^{\text {ST }}$ Century - A Risk-Based Approach Final Report. September 2004a. Disponível em: http://www.fda.gov/ downloads/Drugs/DevelopmentApprovalProcess/Manufacturing/QuestionsandAnswersonCurrentGoodManufacturingPracticescGMPforDrugs/UCM176374.pdf. Acesso em: 16 nov 2016.

8. CVM. Department of Health and Human Services. Food and Drug Administration. Center for Drug Evaluation and Research (CDER). Center for Veterinary Medicine (CVM). Office of Regulatory Affairs (ORA). Guidance for Industry PAT - A Framework for Innovative Pharmaceutical Development, Manufacturing, and Quality Assurance. September 2004b. Disponível em: http://www.fda. gov/downloads/Drugs/.../Guidances/ucm070305.pdf. Acesso em: 11 nov 2016.

9. ICH. International Conference On Harmonisation Of Technical Requirements for Registration of Pharmaceuticals for Human Use. ICH Harmonised Tripartite Guideline. Pharmaceutical Development Q8(R2). August 2009. Disponível em: http://www.ich.org/fileadmin/ Public_Web_Site/ICH_Products/Guidelines/Quality/ Q8_R1/Step4/Q8_R2_Guideline.pdf. Acesso em: 16 nov 2016 .

10. ICH. International Conference on Harmonisation of Technical Requirements for Registration of Pharmaceuticals for Human Use. ICH Harmonised Tripartite Guideline. Development and Manufacture of Drug Substances (Chemical Entities and Biotechnological/Biological Entities) Q11. 1 May 2012. Disponível em: http://www.ich. org/fileadmin/Public_Web_Site/ICH_Products/Guidelines/Quality/Q11/Q11_Step_4.pdf. Acesso em: 17 nov 2016.

11. Yu L, Amidon G, Khan M, Hoag S, Polli J, Raju G, Woodcock J. Understanding Pharmaceutical Quality by Design. AAPS J. 2014; 16(4):771-783. DOI: 10.1208/ s12248-014-9598-3.

12. Charoo NA, Shamsher AAA, Zidan AS, Rahman Z. Quality by design approach for formulation development: A case study of dispersible tablets. Int J Pharm. 2012; 423(2):167-178. DOI: 10.1016/j.ijpharm.2011.12.024.

13. Van Buskirk GA, Asotra S, Balducci C, Basu P, Didonato G, Dorantes A, Eickhoff W, Ghosh T, González M, Henry T, et al. Best Practices for the Development, Scale-up, and Post-approval Change Control of IR and MR Dosage Forms in the Current Quality-by-Design Paradigm. AAPS PharmSciTech. 2014; 15(3):665-693. DOI: 10.1208/s12249-014-0087-x.
14. Yu LX. Pharmaceutical quality by design: product and process development, understanding and control. Pharmaceut Res. 2008; 25(4):781-91. DOI: 10.1007/s11095007-9511-1.

15. Yu LX, Woodcock J. FDA Pharmaceutical Quality Oversight. Int J Pharm. 2015; 491(1):2-7. DOI: 10.1016/j.ijpharm.2015.05.066.

16. De Beer T, Burggraeve A, Fonteyne M, Saerens L, Remon JP, Vervaet C. Near infrared and Raman spectroscopy for the in process monitoring of pharmaceutical production processes. Int J Pharm. 2010; 417(1-2):32-47. DOI: 10.1016/j.ijpharm.2010.12.012.

17. Potter C. PQLI Application of Science- and Risk-based Approaches (ICH Q8, Q9, and Q10) to Existing Products. J. Pharm Innov. 2009; 4(1):4-23. DOI: /10.1007/ s12247-009-9051-9.

18. Lourenço V, Lochmann D, Reich G, Menezes JC, Herdling T, Schewitz J. A quality by design study applied to an industrial pharmaceutical fluid bed granulation. Eur J Pharm Biopharm. 2012; 81(2):438-447. DOI: 10.1016/j. ejpb.2012.03.003.

19. Stocker E, Toschkoff G, Sacher S, Khinast JG. Use of mechanistic simulations as a quantitative risk-ranking tool within the quality by design framework. Int J Pharm. 2014; 475(1-2):245-255. DOI: 10.1016/j.ijpharm.2014.08.055.

20. Badawi MA, El-Khordagui LK. A quality by design approach to optimization of emulsions for electrospinning using factorial and D-optimal designs. Eur J Pharmaceut Sci. 2014; 16(58):44-54. DOI: 10.1016/j. ejps.2014.03.004.

21. Xu X, Al-Ghabeish M, Rahman Z, Krishnaiah YSR, Yerlikaya F, Yang Y, Manda P, Hunt RL, Khan MA. Formulation and process factors influencing product quality and in vitro performance of ophthalmic ointments: Int J Pharm. 2015; 493(1-2):412-425. DOI: 10.1016/j.ijpharm.2015.07.066.

22. ICH. International Conference On Harmonisation Of Technical Requirements for Registration of Pharmaceuticals for Human Use. ICH Harmonised Tripartite Guideline. Quality Risk Management Q9. 09 November 2005. Disponível em: http://www.ich.org/fileadmin/ Public_Web_Site/ICH_Products/Guidelines/Quality/Q9/ Step4/Q9_Guideline.pdf. Acesso em: 18 nov 2016

23. Hubert C, Lebrun P, Houari S, Ziemons E, Rozet E, Hubert P. Improvement of a stability-indicating method by Quality-by-Design versus Quality-by-Testing: A case of a learning process. J Pharm Biomed Anal. 2014; 88:401409. DOI: 10.1016/j.jpba.2013.09.026.

24. Visser JC, Dohmen WMC, Hinrichs WLJ, Breitkreutz J, Frijlink HW, Woerdenbag HJ. Quality by design approach for optimizing the formulation and physical properties of extemporaneously prepared orodispersible films. Int J Pharm. 2015; 485(1-2):70-76. DOI: 10.1016/j.ijpharm.2015.03.005. 
25. FDA. Department of Health and Human Services. Food and Drug Administration. Center for Drug Evaluation and Research (CDER). Quality by Design for ANDAs: An Example for Immediate-Release Dosage Forms. Pharmaceutical Development. Report Example QbD for IR Generic Drugs. April 2012. Disponível: http://www. fda.gov/downloads/Drugs/DevelopmentApprovalProcess/HowDrugsareDevelopedandApproved/Approva1Applications/AbbreviatedNewDrugApplicationANDAGenerics/UCM304305.pdf. Acesso em: 11 nov 2016

26. Chavez PF, Lebrun P, Sacré PY, De Bleye C, Netchacovitch L, Cuypers S, Mantanus J, Motte H, Schubert M, Evrard B, Hubert P, Ziemons E. Optimization of a pharmaceutical tablet formulation based on a design space approach and using vibrational spectroscopy as PAT tool. Int J Pharm. 2015; 486(1-2):13-20. DOI: 10.1016/j. ijpharm.2015.03.025.

27. Liew CV, Karande AD, Heng PWS. In-line quantification of drug and excipients in cohesive powder blends by near infrared spectroscopy. Int J Pharm. 2010; 386(1):138148. DOI: 10.1016/j.ijpharm.2009.11.011.

28. Sarraguça MC, Ribeiro RS, Santos AO, Silva MCD, Lopes JA. A PAT approach for the on-line monitoring of pharmaceuticalco-crystals formation with near infrared spectroscopy. Int J Pharm. 2014; 471(1-2): 478-484. DOI: 10.1016/j.ijpharm.2014.06.003.

29. Jamrógiewicz M. Application of the near-infrared spectroscopy in the Pharmaceutical technology. J Pharm Biomed. 2012; 66:1-10. DOI: 10.1016/j.jpba.2012.03.009.

30. Fonteyne M, Vercruysse J, De Leersnyder F, Van Snick B, Vervaet C, Remon JP, De Beer T. Process Analytical Technology for continuous manufacturing of solid-dosa- ge forms. TrAC Trends in Anal Chem. 2015; 67:159-166. DOI: 10.1016/j.trac.2015.01.011.

31. Tomba E, Facco P, Bezzo F, Barolo M. Latent variable modeling to assist the implementation of Quality-by-Design paradigms in pharmaceutical development and manufacturing: A review. Int J Pharm. 2013; 457(1):283297. DOI: 10.1016/j.ijpharm.2013.08.074.

32. Drakulich A. Real time release testing: industry and regulatory experts discuss the challenges and benefits of implementing real timerelease testing in a pharmaceutical manufacturing environment. (Process Analytical Technology) (Cover story) Pharm Technol. 2011; 35(2):42-46.

33. Escotet-Espinoza MS, Singh R, Sen M, O’Connor, T, Lee S, Chatterjee S, Ramachandran R, Ierapetritou M, Muzzio FJ. Flowsheet models modernize pharmaceutical manufacturing design and risk assessment: in silico, design facilitates process optimization and evaluation of process control strategies. Pharm Technol. 2015; 39:1-6.

34. Nasr MM, Winkle HN. FDA Perspectives: Understanding Challenges to Quality by Design. Directors from FDA's Center for Drug Evaluation and Research summarize findings in an FDA-commissioned report on $\mathrm{QbD}$ and propose actions the agency can take to encourage full-scale QbD implementation. Pharm Technol. 2011; 35(9).

35. ICH. International Conference on Harmonisation of Technical Requirements for Registration of Pharmaceuticals for Human Use. Quality Implementation Working Group on Q8, Q9 and Q10 Questions \& Answers (R4). 11 November 2010. Disponível em: http://www.ich. org/fileadmin/Public_Web_Site/ICH_Products/Guidelines/Quality/Q8_9_10_QAs/Q-IWG_Q_A_R4_Step4_ Nov.2010.pdf. Acesso em: 17 nov 2016. 